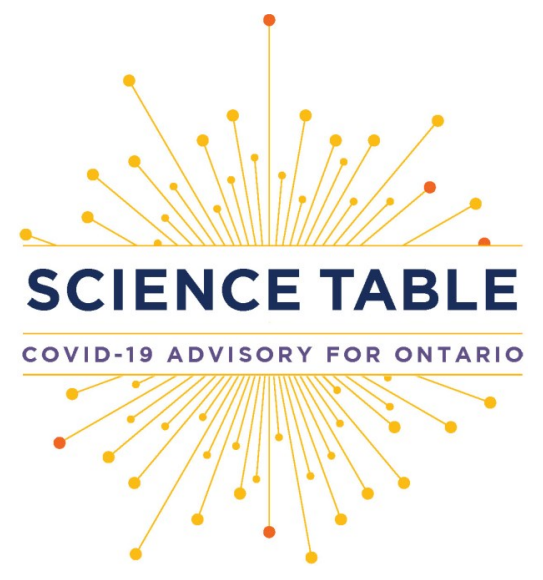

Version 1.0

Published: February 1, 2021

Citation: Choi Y, Stall NM, Maltsev A, et al. Lessons Learned from Israel's Vaccine Rollout. Science Briefs of the Ontario COVID19 Science Advisory Table. 2021;2(9). https:// doi.org/10.47326/ocsat.2021.02.09.1.0

Author Affiliations: The affiliations of the members of the Ontario COVID-19 Science Advisory Table can be found at https:// covid19-sciencetable.ca/.

Declarations of Interest: The declarations of interest of the members of the Ontario COVID-19 Science Advisory Table can be found at https://covid19-sciencetable.ca/.

About Us: The Ontario COVID-19 Science Advisory Table is a group of scientific experts and health system leaders who evaluate and report on emerging evidence relevant to the COVID-19 pandemic, to inform Ontario's response. Our mandate is to provide weekly summaries of relevant scientific evidence for the COVID-19 Health Command Table of the Province of Ontario, integrating information from existing scientific tables, Ontario's universities and agencies, and the best global evidence. The Science Table summarizes its findings for the Health Command Table and the public in Science Briefs.

Correspondence to: Secretariat of the Ontario COVID-19 Science Advisory Table (info@covid19-sciencetable.ca)

Copyright: 2021 Ontario COVID-19 Science Advisory Table. This is an open access document distributed under the terms of the Creative Commons Attribution License, which permits unrestricted use, distribution, and reproduction in any medium, provided that the original work is properly cited.

The views and findings expressed in this Science Brief are those of the authors and do not necessarily reflect the views of all of the members of the Ontario COVID-19 Science Advisory Table and its partners.

\title{
Lessons Learned from Israel's Vaccine Rollout
}

Yoojin Choi, Nathan M. Stall, Antonina Maltsev, Chaim M. Bell, Isaac I. Bogoch, Tal Brosh, Gerald A. Evans, Allan Grill, Jessica Hopkins, David M. Kaplan, Allison McGeer, Jacob Moran-Gilad, Dominik Nowak, Justin Presseau, Asher Salmon, Brian Schwartz, Peter Jüni on behalf of the Ontario COVID-19 Science Advisory Table

\section{Key Message}

As Ontario expands access to the COVID-19 vaccine beyond the Phase 1 priority populations, strategic planning and execution of mass vaccine rollout will have a significant impact on the health and safety of Ontario's 14.5 million residents.

There are six key elements of Israel's successful COVID-19 vaccine campaign that can be readily applied to Ontario to expedite and expand the province's vaccine rollout strategy: a simple vaccine prioritization process; modification to the transport, storage, and distribution of the vaccines; effective communication to promote vaccine confidence; decentralization of vaccination sites; centralized organization through Health Maintenance Organizations (HMOs) using a fully integrated information technology (IT) system in a universal health care system; and the engagement of community-based personnel, infrastructure, and resources.

\section{Background}

Ontario's COVID-19 vaccine rollout is currently underway, with over 265,000 doses administered as of January 21, 2021. This number reflects the targeted deployment of vaccines to the province's Phase 1 priority populations of the rollout plan. This includes long-term care (LTC) residents and staff, hospital healthcare workers, Indigenous communities, and essential caregivers. ${ }^{1}$

As Ontario expands and accelerates access to the COVID-19 vaccine in the coming months, strategic planning and execution of mass vaccine rollout will have a significant impact on the health and safety of Ontario's 14.5 million residents, as well as movement toward a post-pandemic Ontario. ${ }^{2}$

As of January 21, 2021, the State of Israel is leading the world with the greatest number of COVID-19 vaccine doses administered per capita, with over 35 doses per 100 persons completed to date. This rate also places them fourth in the world for the absolute number of administered COVID-19 vaccines. ${ }^{3}$ Understanding the strengths of Israel's campaign and learning from their experience can help optimize Ontario's COVID-19 vaccine rollout. In fact, it was reported that Premier Doug Ford and Health Minister Christine Elliott have already communicated with Israeli officials with regards to vaccine rollout. ${ }^{4,5}$

\section{Questions}

What are the key elements driving the success of Israel's COVID-19 vaccine rollout strategy?

Can these elements be applied or adapted to Ontario's vaccine rollout strategy? 


\section{Findings}

There are six elements in the implementation of Israel's COVID-19 vaccine rollout strategy that can be readily applied or adapted to Ontario to expedite and expand the province's vaccine rollout strategy.

\section{Elements of Israel's COVID-19 Vaccine Rollout Strategy That Are Likely to Be Effective in Ontario}

1. Simple prioritization and vaccinating as fast as possible to reduce morbidity and mortality

2. Modifications to the manufacturer's initial requirements for vaccine transport, storage, and distribution

3. Efficient communication strategies with messaging tailored to specific subgroups to promote vaccine confidence

4. Multiple decentralized vaccination sites to enhance access

5. Centralized organization through the four nationally established HMOs using a fully integrated IT system in a universal health care system

6. Leveraging community-based clinicians, infrastructure, and resources

Simple Prioritization and Vaccinating as Fast as Possible to Reduce Morbidity and Mortality

Similar to Ontario, older people experienced a disproportionately high burden of COVID-related deaths in Israel. ${ }^{6}$ To address this disparity, Israel relied on a simple vaccine prioritization process, with age being the only determining factor, with the exception of healthcare providers and first responders. Having already vaccinated a large proportion of the population aged 60 years and above, Israel is seeing early signs of reduction in critically ill individuals in this age group. ${ }^{7}$ Israel has now expanded to vaccinating individuals over the age of 50 years. Since age is the single greatest risk factor for COVID-19 mortality, simplified distribution plans to maximize upfront administration of vaccine to older adults could avoid unintentionally prioritizing other groups who are at lower risk of death. ${ }^{8}$

\section{Modifications to Vaccine Transport, Storage, and Distribution}

One of the key technical challenges with Pfizer-BioNTech's vaccine is the manufacturer's requirement to transport all 975 doses, which are contained in 195 vials, together in large trays after their removal from freezers set to $-70^{\circ} \mathrm{C}$. This technical specification poses a challenge for vaccinating individuals in smaller settings, for example, in LTC and retirement homes, or in rural locations. 


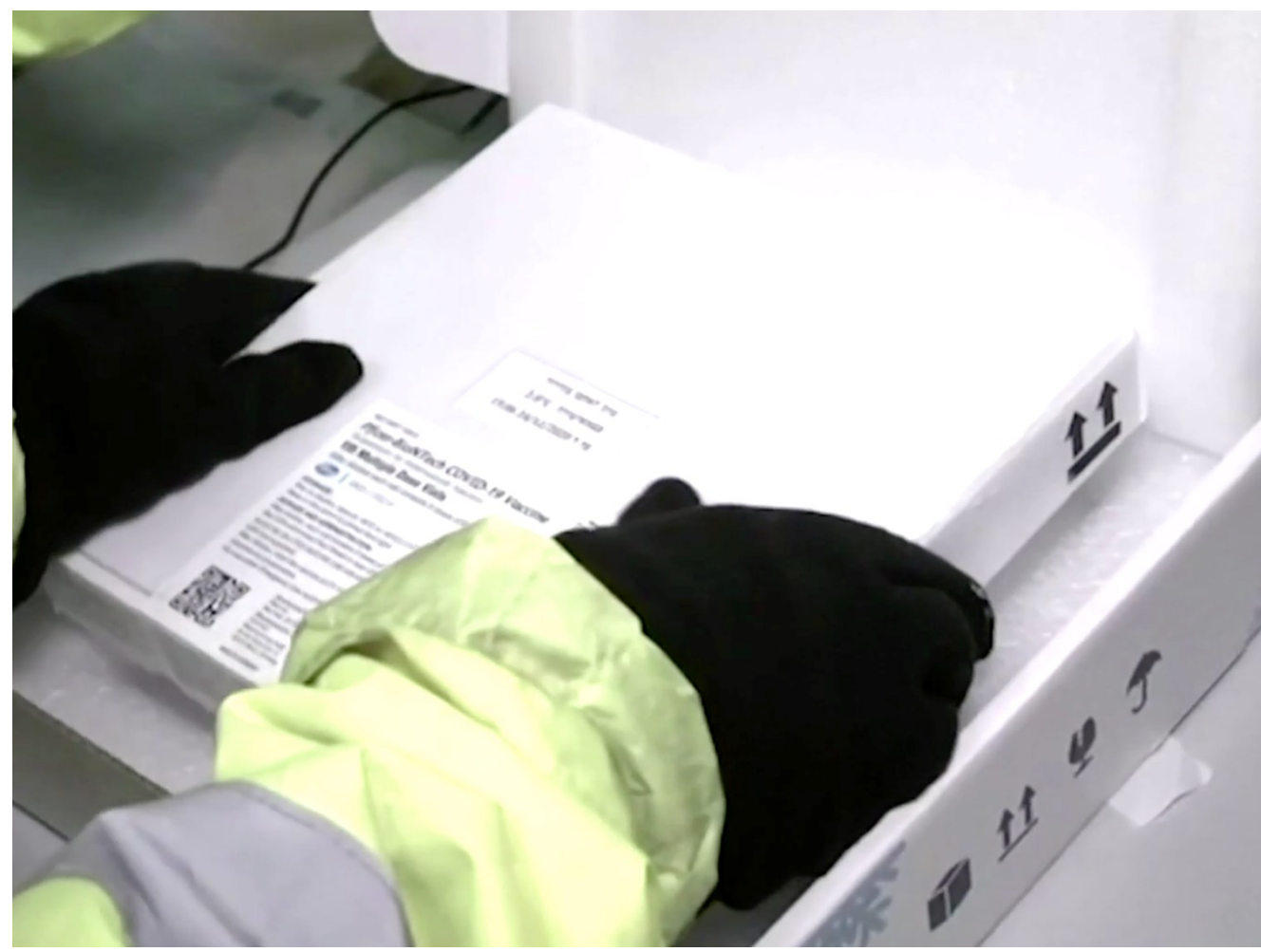

Figure 1. COVID-19 Vaccines Repacked into Smaller Boxes in Israel with the Approval from Pfizer

To solve this issue, Israeli teams have received approval from Pfizer and "repacked the large ultra-frozen pallets into insulated boxes the size of small pizza boxes, allowing for distribution in smaller numbers and at more remote sites" (Figures 1 and 2). ${ }^{9}$

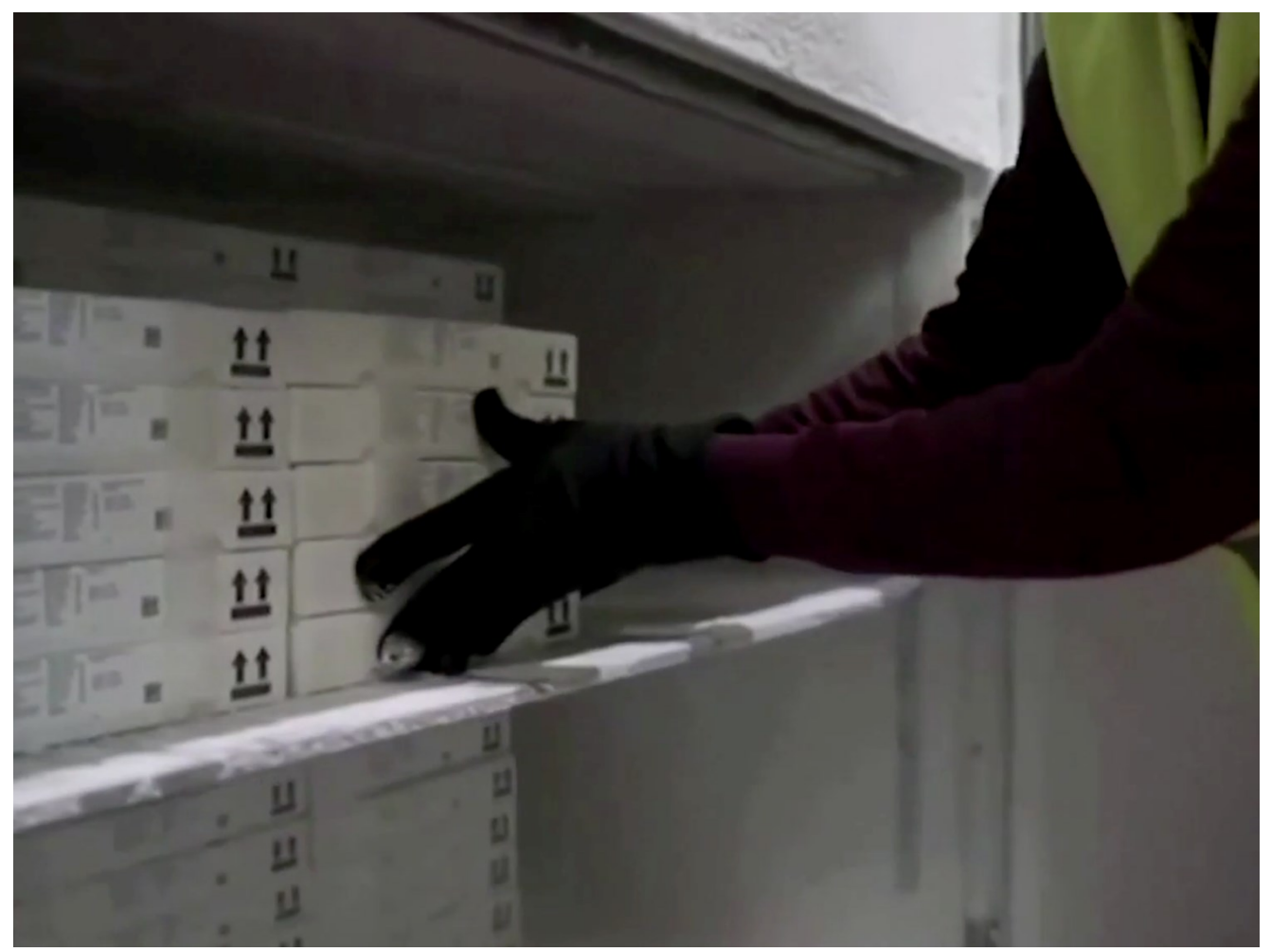

Figure 2. Repacked Vaccines Stored at $-70^{\circ} \mathrm{C}$ Prior to Distribution in Israel

The boxes can currently contain either 50 vials, which corresponds to approximately 300 doses (Figure 3) or 10 vials, which corresponds to approximately 60 doses. The vaccines now arrive at several national centres operated by Ministry of Health 
subcontractors where they are stored at $-70^{\circ} \mathrm{C}$ and repackaged in small boxes to be shipped locally three times a week.

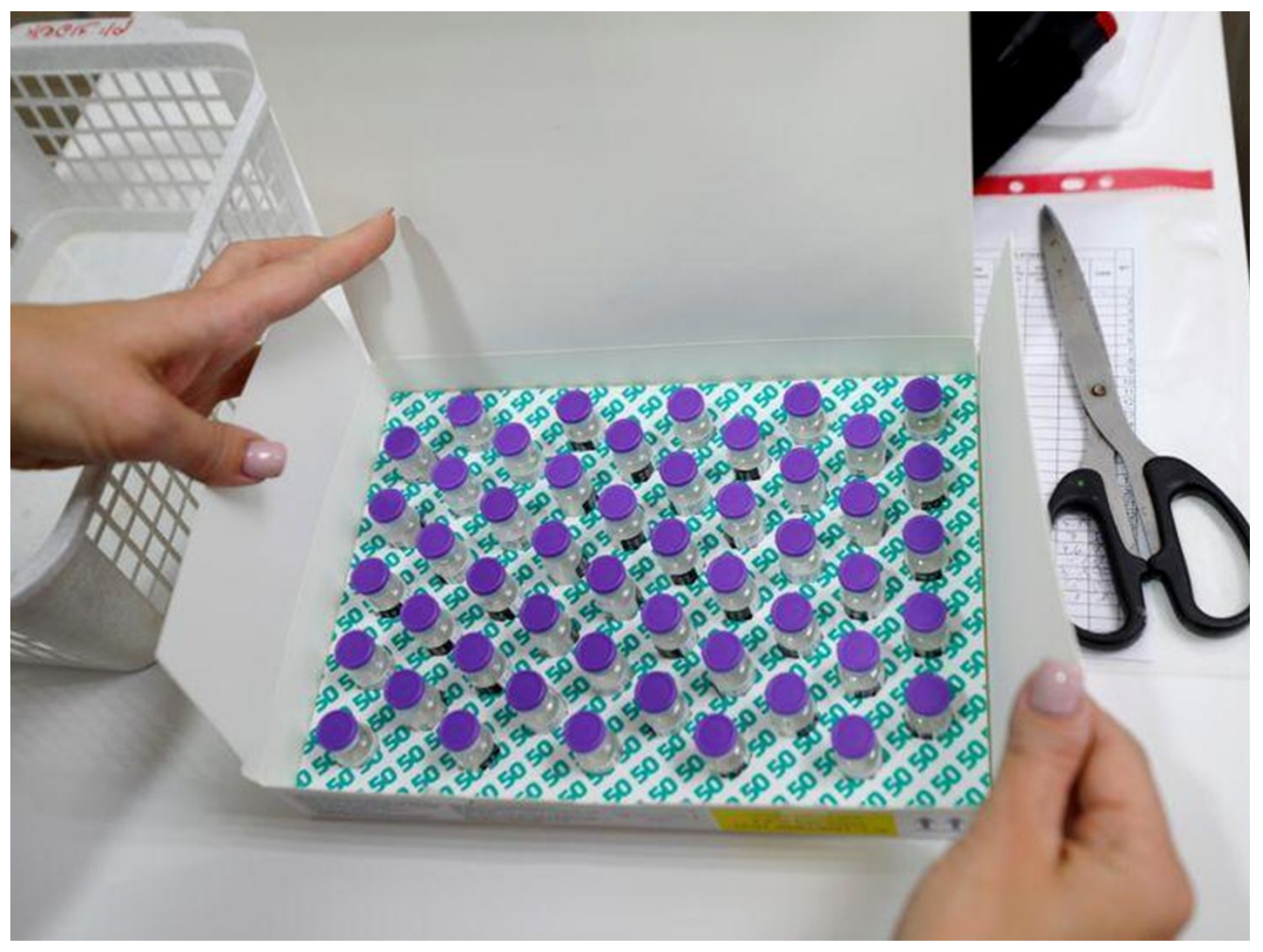

Figure 3. The Inside of a Repacked Box of COVID-19 Vaccine in Israel, Containing 50 Vials of the Vaccine

Similarly, Ontario would benefit from receiving approval from vaccine manufacturers to repackage and transport smaller quantities to specific settings such as LTC homes, retirement homes, Indigenous communities, and rural communities without compromising the quality of the vaccines. This modified strategy can ensure that distribution of vaccines is first received by populations at highest risk of death from COVID-19 with minimal waste of vaccine doses.

\section{Communication Strategies and Tailored Messaging to Promote Vaccine Confidence}

Israel used multiple communication and outreach strategies - social media, organizational websites, and mass media - to enhance reach and promote vaccine confidence in different populations. These efforts included providing daily updates on the number of vaccinated individuals via mass media and addressing antivaccination messages on social media. Tailored messaging is also important, as it is normal for people to mistrust government or institutions for a variety of reasons or based on their perception of the jurisdiction's pandemic response. Importantly, the Israeli Ministry of Health also secured endorsements from religious leaders who in turn helped encourage religious populations such as Ultra-Orthodox Jews and Muslims to get vaccinated.

Using public endorsements from trusted leaders may be an effective way to increase vaccine acceptance. In Israel, Prime Minister Benjamin Netanyahu, in a nationally televised event, was the first to receive a COVID-19 vaccine. Former U.S. presidents have previously announced their intent to get vaccinated for this same reason as well. ${ }^{10}$ Likewise, Ontario could use multiple media outlets to reach diverse populations and work closely with trusted community leaders, trusted individuals such as social media influencers, trusted health professionals such as family doctors, and trusted sources in personal social networks to further promote vaccine confidence. ${ }^{11,12}$ 


\section{Multiple Decentralized Vaccination Sites to Enhance Access}

Decentralized vaccination sites contributed significantly to the rapid rollout of the Israeli COVID-19 vaccination program by increasing reach and access. In addition to hospitals and pharmacy chains, Israel created pop-up and drive-thru vaccination centres (Figures 4 to 6 ) to increase efficiency and accessibility of vaccination. Potential vaccine recipients were required to fill and sign a paper-based questionnaire to determine their eligibility to receive a COVID-19 vaccine. Filling and signing the questionnaire, and receiving the vaccine, were considered as implied consent, without necessitating a formal written consent process.

All subsequent documentation, such as adverse event reporting, took place electronically, and the elimination of paper records helped further expedite the process. Vaccine recipients were monitored for at least 15 minutes after vaccination for potential signs of adverse events; those with a previous history of anaphylaxis were observed for 30 minutes.

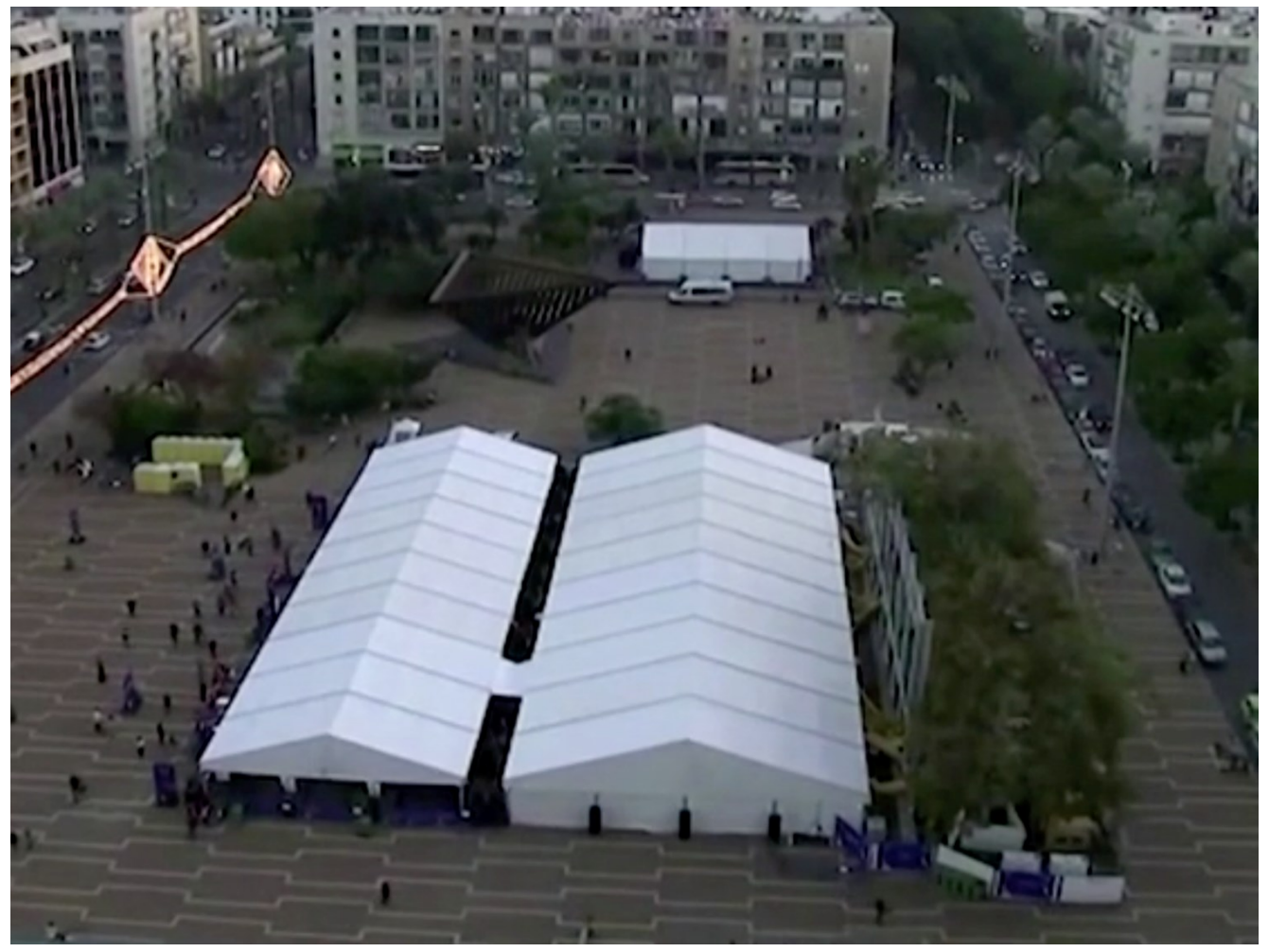

Figure 4. A Pop-up Vaccination Center in Israel

In the case of drive-thru centres (Figure 6), the driver and other passengers were vaccinated all at once by putting their arms out the window of the vehicle. 


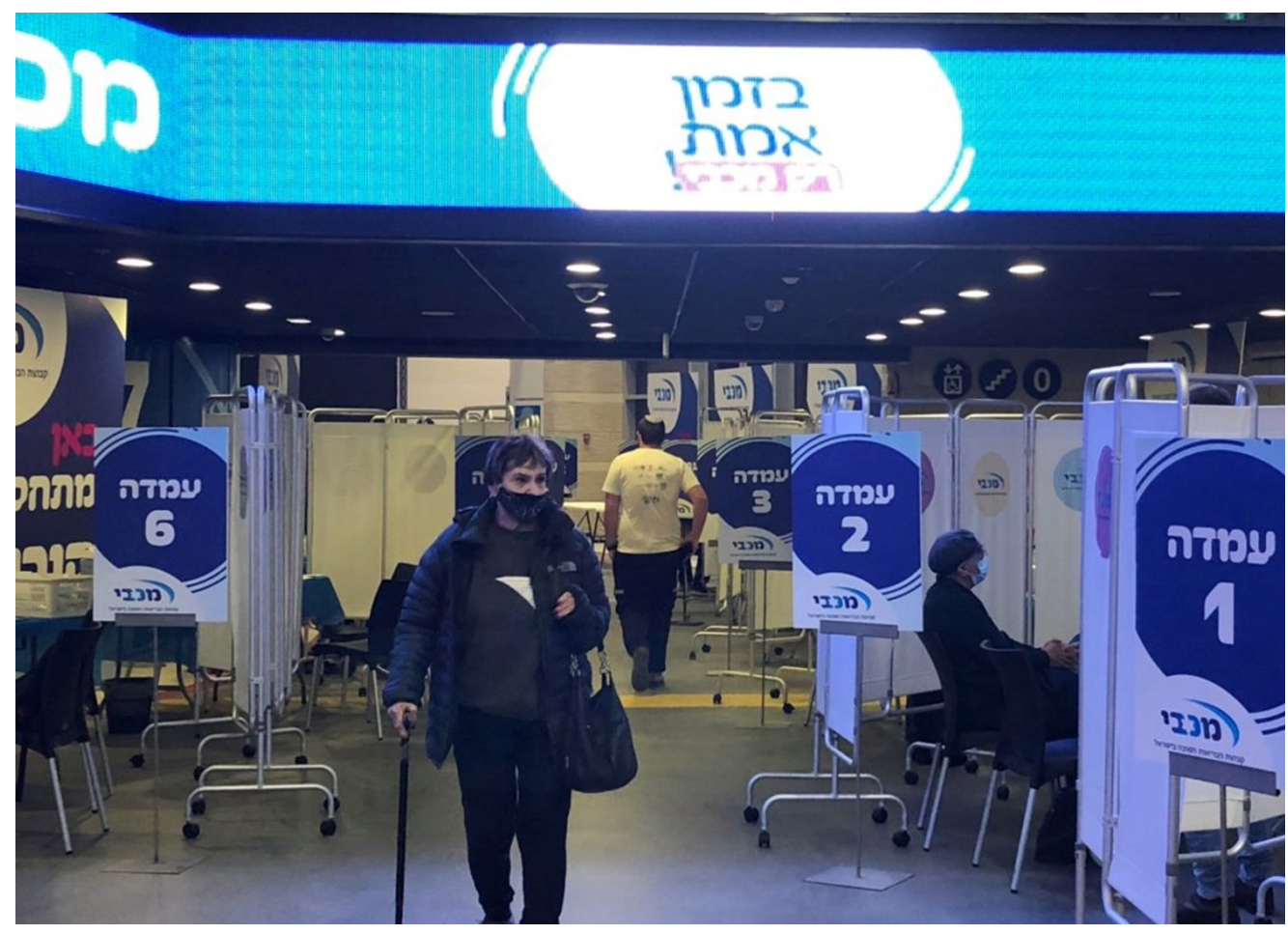

Figure 5. The Inside of a Pop-up Vaccination Center in Israel

Throughout the rollout, decentralized vaccination sites could be added in Ontario to ensure equity in vaccination rates. Many communities might not be able to or willing to access vaccination hubs. Mobile teams will be needed for homebound older adults, shelters, and other congregate settings. Community-tailored programs will also be important, to serve groups who may not otherwise have equitable vaccination rates or protection from COVID-19. Implied, rather than formal written consent could be considered for COVID-19 vaccines in Ontario in the future.

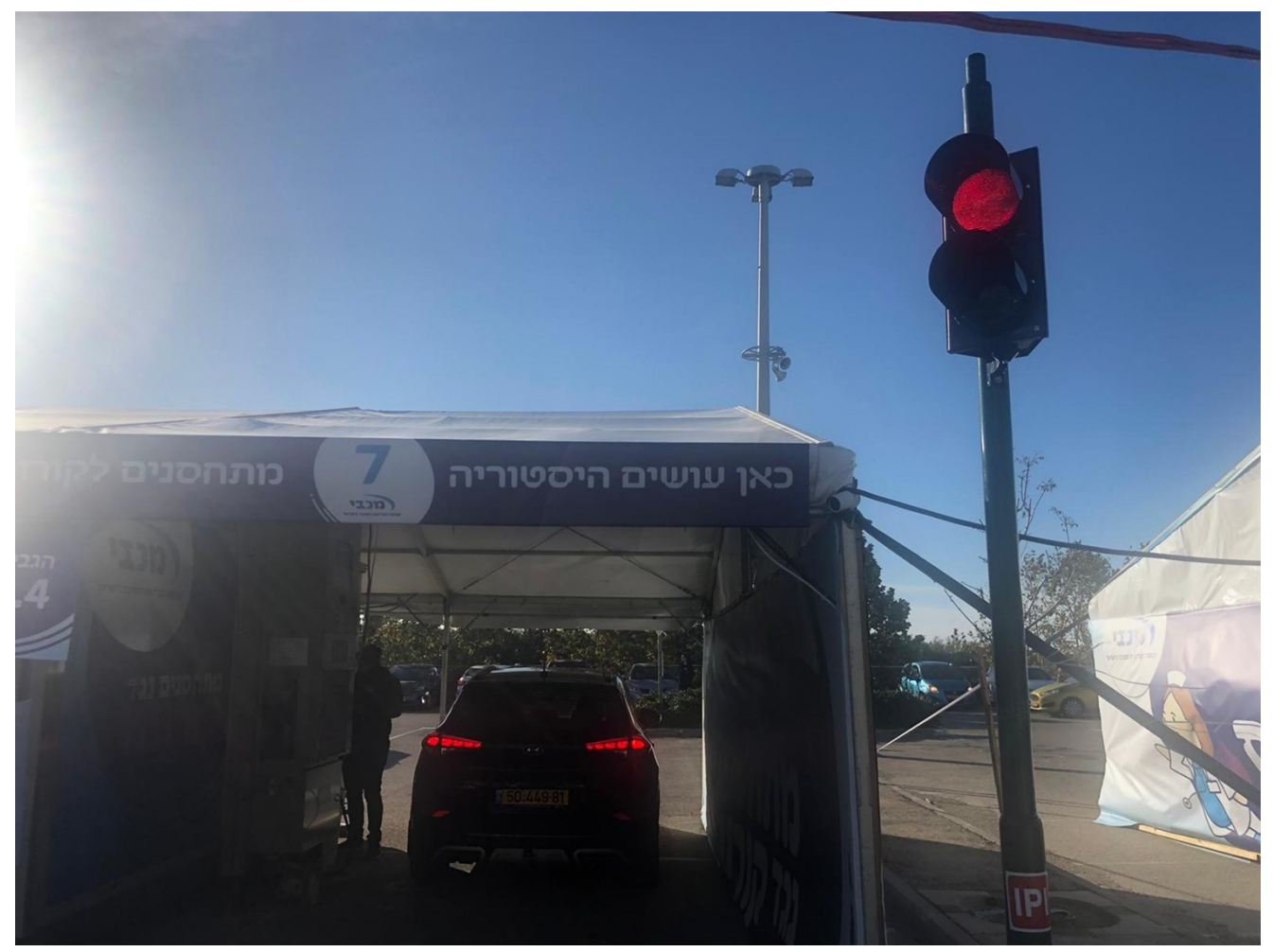

Figure 6. A Drive-Thru Vaccination Center in Israel 


\section{Centralized Organization Through the 4 Established HMOs Using a Fully Integrated} IT System in a Universal Health Care System

Israel leveraged strong organizational and technological capacities to streamline vaccination processes. Like Ontario, Israel has a universal health care system. This allowed a centralized organization through the four established HMOs that cover the entire Israeli population using a fully integrated IT system.

Israel uses a number of different ways to schedule a vaccination including call centres, a health plan website and a mobile application. Shortly after booking an appointment, people received reminders about their vaccination appointments, and when to return for the second dose, through text messages. Importantly, vaccinated individuals subsequently received text messages encouraging them to report adverse events, which created a sense of trust in the system and transparency with the vaccination process. This simple and highly accessible method of reporting adverse events in turn facilitated rapid data collection for a national registry of adverse vaccine events.

Communication was also streamlined for professionals administering vaccines who could phone the call centre on a $24 / 7$ basis for administration guidance, clinical questions, and shipment information. The availability of additional vaccine doses due to overstock was communicated locally and the overstock was administered to local individuals irrespective of their age eligibility. This support at the front line helped ensure that implementation is optimized on the fly and that all doses are maximally used.

Ontario can also benefit from using technology to streamline communication with healthcare professionals as well as the general public; an effective IT system can make vaccination simple for everyone and help build trust and transparency in the system.

\section{Leveraging Community-Based Clinicians, Infrastructure, and Resources}

Israel took advantage of community-based nurses, physicians, trained emergency medical technicians (EMTs) and paramedics to help rapidly administer the COVID-19 vaccine. Notably, one-third of the country's nurses work in community settings, many of whom are experienced in vaccine administration. ${ }^{13}$

In the context of Ontario, leveraging primary care providers, including family doctors, nurse practitioners and other community-based clinicians who have established relationships with patients is key to promoting vaccine confidence, while increasing human resources required to administer vaccines as quickly as the supply will allow. Family doctors and primary care professionals administer the majority of flu vaccinations each year in Ontario and can identify and counsel priority individuals in their practices.

\section{Interpretation}

There are several key differences between Israel and Ontario with respect to geography, the system of government, healthcare system, and existing infrastructure that precludes applying some of the elements of Israel's COVID-19 vaccine campaign to Ontario. Key caveats to consider when interpreting Israel's experience include Israel's highly dense population of 9.5 million people in a small geographic area with a generally younger population in comparison to Canada, and Israel's experience in running large-scale national emergency operations. Nevertheless, the aforementioned six key elements of Israel's successful COVID-19 vaccine campaign may be adapted or directly implemented to optimize Ontario's COVID-19 vaccine rollout. 


\section{Author Contributions}

YC and NS wrote the first draft of the Science Brief. All authors contributed to the conception of the Science Brief, revised it critically for important intellectual content, and approved the final version.

\section{References}

1. Government of Ontario. COVID-19 vaccines for Ontario. COVID-19 in Ontario. Published 2021. Accessed January 21, 2021. https://covid-19.ontario.ca/covid-19vaccines-ontario

2. Government of Ontario. Ontario demographic quarterly: highlights of third quarter 2020. Ontario.ca. Published December 15, 2020. Accessed January 21, 2021. https://www.ontario.ca/page/ontario-demographic-quarterly-highlights-thirdquarter-2020

3. Hannah R, Ortiz-Ospina E, Beltekian D, et al. Coronavirus (COVID-19) vaccinations - statistics and research. Our World in Data. Published January 21, 2021. Accessed January 21, 2021. https://ourworldindata.org/covid-vaccinations

4. Mulligan C. NEW: Premier Ford begins news conference by saying he has spoken with Israeli officials to discuss vaccine rollout - it has been lauded for it's quick immunization program. \#covid19. Published January 25, 2021. Accessed January 25, 2021. https://twitter.com/CityCynthia/status/1353765379051577346

5. Helmer A. COVID-19: Ontario to "accelerate" vaccinations for most vulnerable; Province reports 1,958 new cases. OTTAWA CITIZEN. https://ottawacitizen.com/ news/covid-19-a-year-after-ontarios-first-case-province-reports-1958-newinfections-and-43-deaths. Published January 26, 2021.

6. Israel Science and Technology Directory. The age distribution and sex of patients who died because of coronavirus (COVID-19) infection. Published December 31, 2020. Accessed January 26, 2021. https://www.science.co.il/medical/coronavirus/ Death-statistics.php

7. KSM - Maccabi Research and Innovation Center - Publishes Insights from RealTime Monitoring of Corona Virus Vaccination Data. Maccabi Health Services Research Center - KSM, KI Institute Accessed January 25, 2021. https://maurice.nl/ wp-content/uploads/2021/01/Isreal-Maccabi.pdf

8. Government of Ontario. COVID-19 vaccination update. Technical Briefing presented at the: January 13, 2021; Ontario. Accessed January 19, 2020. https:// files.ontario.ca/moh-covid-19-vaccine-technical-briefing-en-january-13-2021-202101-13.pdf

9. Rabinovitch $A$, Lubell $M$, Scheer S. Pizza-sized boxes and paying a premium: Israel's COVID-19 vaccine rollout. Reuters. https://www.reuters.com/article/ushealth-coronavirus-israel-vaccination-idUSKBN29BOKJ. Published January 6, 2021. Accessed January 21, 2021.

10. Volpp KG, Loewenstein G, Buttenheim AM. Behaviorally informed strategies for a national COVID-19 vaccine promotion program. JAMA. 2021;325(2):125-126. https://doi.org/10.1001/jama.2020.24036

11. Ontario reports 2,662 new COVID-19 cases, 87 more deaths. CBC News. https:// www.cbc.ca/news/canada/toronto/covid-19-ontario-janauary-22-2021-stay-athome-1.5883621. Published January 22, 2021. Accessed January 22, 2021.

12. WHO Technical Advisory Group of Behavioural Insights and Sciences for Health. 
Behavioural Considerations for Acceptance and Uptake of COVID-19 Vaccines. World Health Organization; 2020. Accessed January 27, 2021. https://www.who.int/ publications-detail-redirect/9789240016927

13. Nissanholtz-Gannot R, Rosen B, Hirschfeld $M$, et al. The changing roles of community nurses: the case of health plan nurses in Israel. Isr J Health Policy Res. 2017;6(1):69. https://doi.org/10.1186/s13584-017-0197-5 